http://jmscr.igmpublication.org/home/ ISSN (e)-2347-176x ISSN (p) 2455-0450 crossref DOI: https://dx.doi.org/10.18535/jmscr/v8i6.97

\author{
Journal Of Medical Science And Clinical Research \\ IGM Publication \\ An official Publication of IGM Publication
}

\title{
Coagulation in the Prognosis of Severe COVID-19
}

\author{
Authors \\ Kallem Sharat Venkat Reddy ${ }^{1}$, Aakunuri Akhil ${ }^{\mathbf{2}}$ \\ 1,2 Pharm-D, Bharat Institute of Technology, Hyderabad, Telangana-500072
}

\begin{abstract}
A relatively high mortality rate of patients with Corona virus disease 2019 is worrying, with hospitals worldwide continuing to admit flood of patients with corona virus disease, the confusing pathogenesis behind this severe mortality rate is slowly being understood. Respiratory involvement in the progression is already known; new inputs show a possible cardiac system involvement with potential involvement of coagulopathy in COVID-19. To be specific disseminated intravascular coagulation (DIC) with high risk of venous thromboembolism. Severity in COVID-19 patients is shown directly related with coagulation in the patients. Also from an analysis in patients in Wuhan-China, it is reported that heparin treatment reduced mortality rate in patients with severe COVID-19. In this article we describe the potential coagulation features of patients with corona virus disease.

Keywords: COVID-19, Corona virus, disseminated intravascular coagulation (DIC), Anticoagulants, Venous thromboembolism, D-dimer.
\end{abstract}

\section{Introduction: $1,2,3$}

Since its outbreak in Wuhan-China in, COVID-19 has spread to all the parts of the world at an unprecedented rate and with unparalleled mortality rate is not showing any signs of stopping any soon. Due to its puzzling pathogenesis, finding an accurate therapy for COVID-19 is the main challenge in front of the whole world. With increasing autopsies of subjects and studies, the pathogenesis and progression from mild to severe is slowly being unraveled. One of the major reports from majority of the studies show that the subjects with severe corona disease are also affected with significant abnormal coagulation namely disseminated intravascular coagulation, which is playing a significant role in the mortality rate in the COVID-19 patients. Incidence of venous thromboembolism among the COVID-19 patients is also higher in Intensive care units. In patients with severe COVID-19, high D-dimer levels and also fibrin degradation product levels were also seen.

\section{Definitions}

1. Disseminated intravascular coagulation: ${ }^{4}$

DIC is a pretty rare but serious condition in which the patient suffers with a condition where abnormal blood clotting throughout the body blood vessels take place, which is caused by any other disease or conditions such as injury or an infection. Here the body's normal clotting process becomes overactive.

Symptoms include: Bleeding, Bruising, Shortness of breath, Low blood pressure, confusion. 
Complications are life threatening and include bleeding or multiple organ failure.

\section{Venous Thromboembolism (VTE): ${ }^{5}$}

VTE is a condition in which a blood clot forms commonly in the deep veins of arms, legs or arms (this condition is called as deep vein thrombosis(DVT)) and travels in the circulation and settles in the lungs (known as pulmonary embolism, PE). Together DVT and PE are known as VTE, which is dangerous and deadly medical condition.

Symptoms include: Pain or tenderness starting in the calf, swelling in ankle or foot, shortness of breath, rapid breathing, chest pain, light headedness or passing out.

\section{D- dimer: ${ }^{6}$}

D-dimer is the fibrin degradation product (FDP), or a small fragment of protein present in the blood after a blood clot is degraded by fibrinolysis.

D-dimer concentration is determined by blood test to diagnose thrombosis and also to diagnose disseminated intravascular coagulation.

Four fold increases in the D-dimer is a strong indicator for the mortality in COVID-19 patients.

\section{Clinical Course of COVID-19: ${ }^{7,8,9}$}

When a person is infected with SARS-CoV-2, the damage is not only limited to lungs. The virus results in the manifestations of symptoms such as fever, diarrhea, fatigue, pharyngalgia ${ }^{\mathbf{A}}$ and other symptoms too. The incubations take 1-14 days (37 days being common). At this phase the blood leukocytes and lymphocytes are not significantly reduced. The virus further spreads in the bloodstream mainly in the lungs, GIT, and also heart, mostly concentrated in the tissues expressing ACE-2, which is the receptor of SARS-CoV-2. This phase occurs after the onset of symptoms that is 7-14 days and a second attack is started by the virus. Now the lesions in the lungs become worse and the peripheral blood leukocytes and lymphocytes reduce significantly including both $\mathrm{B}$ and $\mathrm{T}$ lymphocytes. Inflammatory factors in the blood are increased.

\section{Abnormal Coagulation Condition: 7, 10,11}

Patients after the above mentioned phase start developing hypercoagulable state and D-Dimer coagulation factors may appear abnormal. During the development of dyspnoea ${ }^{\mathbf{B}}$ D-Dimer increased from mild to significant also prolonged prothrombin time (PT) and also gradual decrease of fibrinogen and platelet was seen. In some non survivor patients of COVID-19 it was observed that they suffered from ecchymosis ${ }^{\mathbf{C}}$ of fingers and toes and also functions of heart and kidneys worsened. These manifestations are consistent with diagnosis of the hypercoagulable phase of disseminated intravascular coagulation. Presumably COVID-19 can activate coagulation through various mechanisms, further leading to severe hypercoagulability.

\section{Venous Thromboembolism and Disseminated Intravascular Coagulation in Covid-19 Patients: 12, 13, 14}

Patients hospitalized for COVID-19 are prone to excessive coagulation activation leading to thrombotic conditions. In the studies recently done from autopsies and other trails it is observed that mortality from COVID-19 was directly proportional with the abnormal coagulation in the patients. Organ dysfunction and coagulopathy were associated with high mortality. In some studies done it was observed that D-Dimer results and fibrin degradation product levels in the blood were significantly elevated in the patients who didn't survive. Also in the patients with severe COVID-19 and in intensive care units, the incidence of DIC and VTE was significantly higher.

D-Dimer might help in the early recognition of this in the high risk patients. Recent recommendations also suggest that all the patients who are admitted with COVID-19 should receive 
thromboprophylaxis, if such an indication is present. Recent data shows that patients treated with anticoagulants were associated with lower mortality rate.

Heparin also LMWH is implicated to be the best choice of anticoagulant for the admitted patients according to preliminary data.

\section{Results and Conclusions from the Studies Done Worldwide}

1. Confirmation of the high cumulative incidence of thrombotic complications in critically ill ICU patients with COVID-19: An updated analysis. ${ }^{15}$

F.A.Klok M.J.H.A.Kruip et al.

In this study total of 184 patients with COVID-19 being treated in the Intensive Care Unit of 33 Dutch hospitals were studied. Out of them total 41 died and 78 were discharged alive. All of these patients were given pharmacological thromboprophylaxis.

Pulmonary embolism was seen in 65 patients. 3 patients showed venous thromboembolic events and the Arterial thrombotic events were seen in 7 patients.

It was observed that most of the patients who didn't survive suffered with PE and other abnormal thrombolytic events. They also said that therapeutic anticoagulation at baseline prevented thrombotic complications.

This study finally advised that all the COVID-19 patients admitted in ICU should be given pharmacological thrombosis prophylaxis.

2. High risk of thrombosis in patients with severe SARS-CoV-2 infection: a multicenter prospective cohort studies ${ }^{16}$

Julie Helms et al.

In this study 150 patients from 4 ICU's from two centres of a French tertiary hospital suffering from COVID-19 were included. Out of these 150 patients sixty four clinically relevant thrombotic complications were found.

29 patients experienced circuit clotting complications.12 patients showed thrombotic occlusions $^{\mathbf{D}}$ of centrifugal pump. More than $95 \%$ of patients had elevated D-Dimer and fibrinogen. 50/57 patients also showed positive lupus anticoagulant. Comparison between COVID-19 and non COVID-19 ARDS (Acute respiratory distress syndrome) patients, showed that COVID19 patients developed more thrombotic complications.

According to this study despite anticoagulation, a number of patients with COVID-19 developed life threatening thrombotic complications. This study says that higher anticoagulation targets than usual should probably be taken into consideration.

3. High incidence of venous thromboembolic events in anticoagulated severe COVID-19 patients $^{17}$

Jean-François Llitjos et al.

In this retrospective study 26 patients from 2 French intensive care units were taken into study. All patients were treated with anticoagulants from admission itself. Out of these patients, 18 patients were diagnosed with VTE.6 patients were diagnosed with pulmonary embolism. In all the patients abnormal D-Dimer and fibrin degradation products were found.

The results of this study supported that routine screening of VTE in COVID-19 patients should be done and early therapeutic anticoagulation should be considered.

4. Abnormal coagulation parameters are associated with poor prognosis in patients with novel corona virus pneumonia 18 Ning Tang et al.

In this study total 183 patients suffering with novel corona virus pneumonia in Tongji hospital, Wuhan, China were studied. By the time of these study 21 patients died, 78 patients have been discharged and rest 84 of the patients remained hospitalized.

The coagulation parameters between the survivors and non survivors were compared and it showed a dynamic change in the D-Dimer and FDP levels which were elevated in non survivors.

This study shows that the existence of DIC in patients with NCP is so common and also abnormal D-Dimer and FDP levels have the 
potential to guide and promote better prognosis and therapy.

\section{Anticoagulant treatment is associated with} decreased mortality in severe coronavirus disease 2019 patients with coagulopathy 14 Ning Tang et al.

In this study from Wuhan, China total 449 students were enrolled out of which 99 were given heparin (mostly LMWH) for 7 days and longer. For first 28 days no difference was seen between heparin users and non users but $28^{\text {th }}$ day mortality rate was less in heparin users when compared to non heparin users. Also in these patients D-Dimer was $>6$ fold of the upper limit of normal.

As per the results of this study, anti coagulant therapy mainly with LMWH showed better results in patients with elevated D-Dimer and in those who met the the sepsis induced coagulopathy criteria.

However as per their conclusion maybe anti coagulant therapy can only be effective in patients who meet SIC or in those with significantly elevated D-Dimer. Benefit of this therapy in unselected patients is unknown.

6. Prevalence of venous thromboembolism in patients with severe novel coronavirus pneumonia ${ }^{19}$

Songping Cui et al.

In this study 81 severe novel corona virus pneumonia patients from ICU of Union hospital, Wuhan, China were enrolled.

Out of the 81 patients 20 patients were diagnosed with VTE out of which, 8 patients died with VTE. However by the time of the results of this study some of the patients were still being treated and clinical outcome may change.

As per results of these results, D-dimer could be effectively used to determine active coagulation and also its levels decreased in the patient body gradually with anticoagulant therapy. Hence Ddimer values may also help monitor the effectiveness of anti-coagulants.

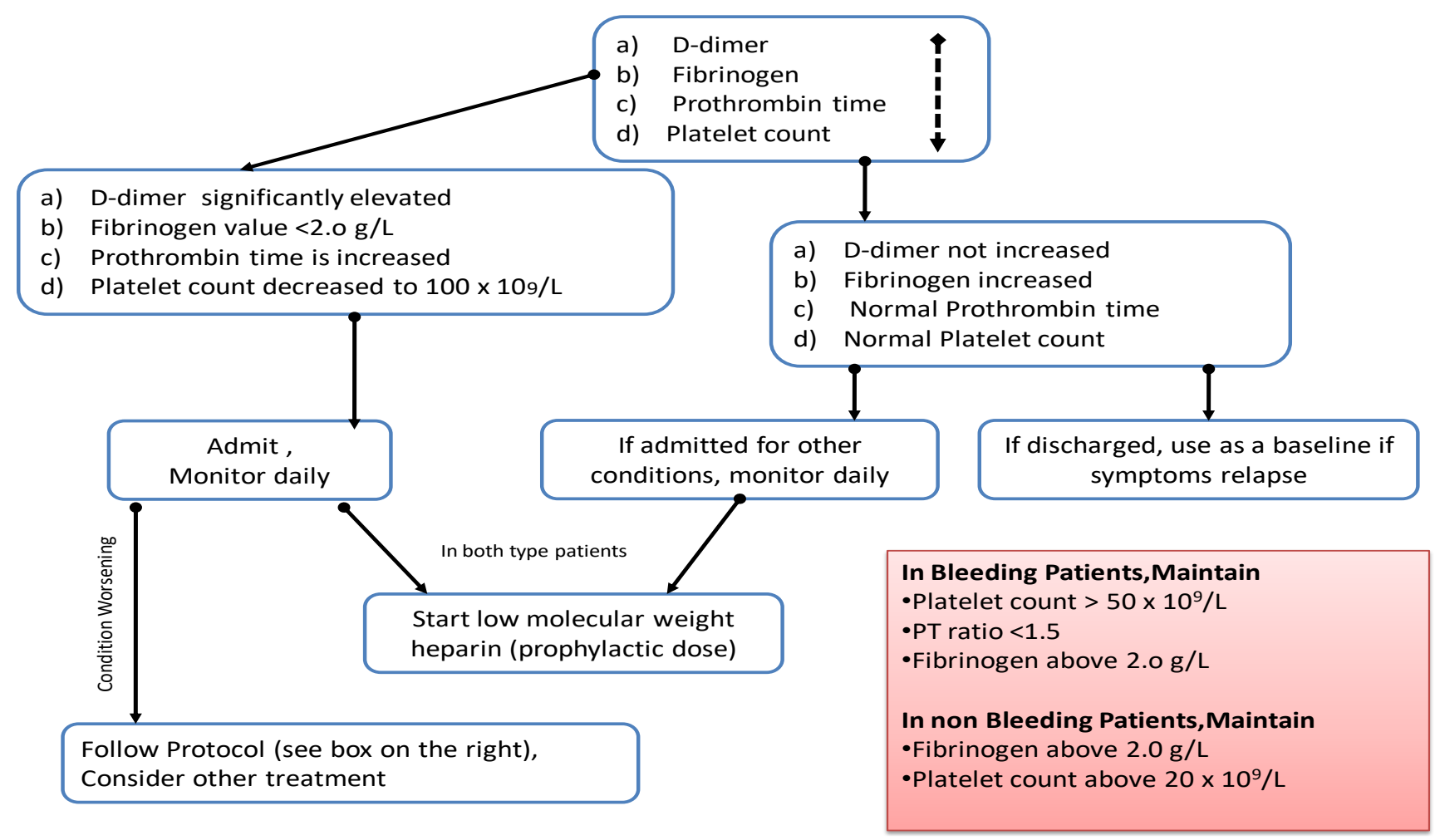

Fig 1 Algorithm for Management of Coagulopathy in COVID-19 21 


\section{Summary}

\section{Management Of Coagulation Issues: ${ }^{3,20}$}

The patients, who are newly hospitalized with COVID-19 or presumptive of COVID-19 infection, should be tested for coagulation along with D-dimer and fibrinogen and also platelet count which can be useful in providing information and prognosis.

The rapid rise in D-dimer and also fibrinogen associated with DIC could be seen within 7-10 days after onset of symptoms or 4-10 days after hospitalization. Also the patients should be treated with pharmacologic VTE prophylaxis starting at the time of admission, apart from if it has any contraindications.

In patients with elevated D-dimer levels at significantly higher levels than the normal level, treatment with Enoxaprin or unfractioned heparin showed decreased mortality rates. Fig 1 . is an algorithm for management of coagulopathy in COVID-19 based on studies.

\section{Conclusion}

While the evidence is rapidly mounting up about COVID-19 associated coagulopathy and risk of thrombosis, there is not much evidence to guide antithrombotic treatment. However results suggest that both screening of coagulopathy and early anticoagulation treatment should be considered in severe COVID-19 patients and also D-dimer levels and FDP should be monitored for the $\operatorname{prognosis}^{\mathbf{E}}$, as VTE and DIC and other thrombotic complications are seen in majority of the non survivors of COVID-19 compared to the survivors. Early treatment with anticoagulants especially LMWH and Enoxaparin have shown to decrease the mortality rate in patients with severe COVID-19 and coagulation. Anyways such high mortality rate of severe COVID-19 is worrying and the treatment plans and suggestions are slowly emerging from the day by day reports and for accurate therapy more prospective studies are needed.
Glossary

\section{A. Pharyngalgia}

Pain in the pharynx.

\section{B. Dyspnoea}

Difficult or labored breathing.

\section{Ecchymosis}

A discoloration of the skin caused due to bleeding underneath, typically caused by bruising.

\section{Occlusions}

Blockage or closing of a blood vessel or a hollow organ.

\section{E. Prognosis}

The course of a medical condition.

\section{References}

1. Porfidia, A., \& Pola, R. (2020). Venous thromboembolism in COVID-19 patients. Journal of Thrombosis and Haemostasis.

2. Tang, N., Li, D., Wang, X., \& Sun, Z. (2020). Abnormal coagulation parameters are associated with poor prognosis in patients with novel coronavirus pneumonia. Journal of thrombosis and haemostasis, 18(4), 844-847.

3. Connors, J. M., \& Levy, J. H. (2020). COVID-19 and its implications for thrombosis and anticoagulation. Blood, The Journal of the American Society of Hematology, 135(23), 2033-2040.

4. Disseminated intravascular coagulation (DIC), National Heart, Lung, and Blood Institute https://www.nhlbi.nih.gov/healthtopics/disseminated-intravascular-coagulation

5. https://www.worldthrombosisday.org/issue/vt e/\#: :text=Venous\%20thromboembolism\%20 (VTE)\%20is\%20a,as\%20pulmonary\%20emb olism\%2C\%20PE).

6. Adam, S. S., Key, N. S., \& Greenberg, C. S. (2009). D-dimer antigen: current concepts and future prospects. Blood, The Journal of the American Society of Hematology, 113(13), 2878-2887. 
7. Li, T., Lu, H., \& Zhang, W. (2020). Clinical observation and management of COVID-19 patients. Emerging microbes \& infections, 9(1), 687-690.

8. Molecular and serological investigation of 2019-nCoV infected patients: implication of multiple shedding routes.Zhang $\mathrm{W}$, Du $\mathrm{RH}$, Li B, Zheng XS, Yang XL, Hu B, Wang YY, Xiao GF, Yan B, Shi ZL, Zhou-P

9. Detectable 2019-nCoV viral RNA in blood is a strong indicator for the further clinical severity.Chen W, Lan Y, Yuan X, Deng X, Li Y, Cai X, Li L, He R, Tan Y, Deng X, Gao M, Tang G, Zhao L, Wang J, Fan Q, Wen C, Tong Y, Tang Y, Hu F, Li F, Tang X.Emerg Microbes Infect. 2020; 9(1):469-473.

10. Hypothesis for potential pathogenesis of SARS-CoV-2 infection-a review of immune changes in patients with viral pneumonia.Lin L, Lu L, Cao W, Li TEmerg Microbes Infect. 2020 Dec; 9(1):727-732.

11. Li, T. (2020). Diagnosis and clinical management of severe acute respiratory syndrome coronavirus 2 (SARS-CoV-2) infection: an operational recommendation of Peking Union Medical College Hospital (V2. 0) working group of 2019 novel coronavirus, Peking union medical college hospital. Emerging microbes \& infections, 9(1), 582585.

12. Atallah, B., Mallah, S. I., \& AlMahmeed, W. (2020). Anticoagulation in COVID-19. European Heart Journal-Cardiovascular Pharmacotherapy.

13. Kollias, A., Kyriakoulis, K. G., Dimakakos, E., Poulakou, G., Stergiou, G. S., \& Syrigos, K. (2020). Thromboembolic risk and anticoagulant therapy in COVID-19 patients: emerging evidence and call for action. British journal of haematology.

14. Tang, N., Bai, H., Chen, X., Gong, J., Li, D., \& Sun, Z. (2020). Anticoagulant treatment is associated with decreased mortality in severe coronavirus disease 2019 patients with coagulopathy. Journal of thrombosis and haemostasis, 18(5), 1094-1099.

15. Klok, F. A., Kruip, M. J. H. A., Van Der Meer, N. J. M., Arbous, M. S., Gommers, D. A. M. P. J., Kant, K. M., ... \& Endeman, H. (2020). Confirmation of the high cumulative incidence of thrombotic complications in critically ill ICU patients with COVID-19: an updated analysis. Thrombosis Research.)

16. Helms, J., Tacquard, C., Severac, F., Leonard-Lorant, I., Ohana, M., Delabranche, X., ... \& Fafi-Kremer, S. (2020). High risk of thrombosis in patients with severe SARSCoV-2 infection: a multicenter prospective cohort study. Intensive care medicine, 1-10.

17. Llitjos, J. F., Leclerc, M., Chochois, C., Monsallier, J. M., Ramakers, M., Auvray, M., \& Merouani, K. (2020). High incidence of venous thromboembolic events in anticoagulated severe COVID-19 patients. Journal of Thrombosis and Haemostasis.

18. Tang, N., Li, D., Wang, X., \& Sun, Z. (2020). Abnormal coagulation parameters are associated with poor prognosis in patients with novel coronavirus pneumonia. Journal of thrombosis and haemostasis, 18(4), 844-847

19. Cui, S., Chen, S., Li, X., Liu, S., \& Wang, F. (2020). Prevalence of venous thromboembolism in patients with severe novel coronavirus pneumonia. Journal of Thrombosis and Haemostasis.

20. Tang, N., Li, D., Wang, X., \& Sun, Z. (2020). Abnormal coagulation parameters are associated with poor prognosis in patients with novel coronavirus pneumonia. Journal of thrombosis and haemostasis, 18(4), 844-847.

21. Salim Rezaie(2020) COVID-19:Thrombosis and Hemoglobin. https://rebelem.com/covid-19-thrombosisand-hemoglobin/ 